\title{
Direct Evidence of Multibubble Sonoluminescence Using Therapeutic Ultrasound and Microbubbles
}

\author{
Estelle Beguin, ${ }^{\dagger}$ Shamit Shrivastava, ${ }^{\dagger}$ (i) Nikolai V. Dezhkunov, ${ }^{\ddagger}$ Anthony P. McHale, ${ }^{*} \S_{\odot}$ \\ John F. Callan, ${ }^{*} \S$ and Eleanor Stride ${ }^{* \dagger \dagger}$ \\ ${ }^{\dagger}$ Department of Engineering Science, Institute of Biomedical Engineering, University of Oxford, Oxford OX3 7DQ, United Kingdom \\ ${ }^{\ddagger}$ BSUIR, P. Brovka Street 6, 220013 Minsk, Belarus \\ ${ }^{\S}$ Biomedical Sciences Research Institute, Ulster University, Coleraine BT52 1SA, United Kingdom
}

Supporting Information

\begin{abstract}
The intense conditions generated in the core of a collapsing bubble have been the subject of intense scrutiny from fields as diverse as marine biology and nuclear fusion. In particular, the phenomenon of sonoluminescence, whereby a collapsing bubble emits light, has received significant attention. Sonoluminescence has been associated predominantly with millimeter-sized bubbles excited at low frequencies and under conditions far removed from those associated with the use of ultrasound in medicine. In this study, however, we demonstrate that sonoluminescence is produced under medically

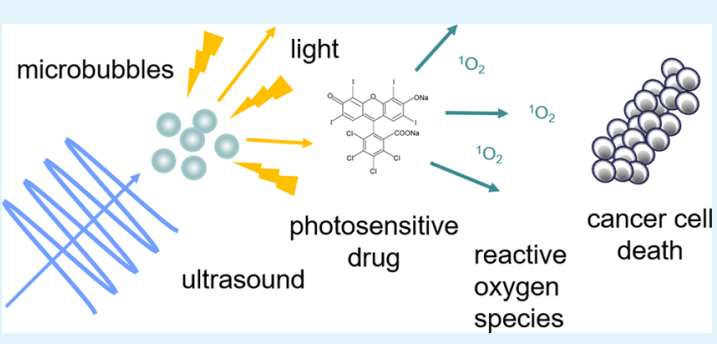
relevant exposure conditions by microbubbles commonly used as contrast agents for ultrasound imaging. This provides a mechanistic explanation for the somewhat controversial reports of "sonodynamic" therapy, in which light-sensitive drugs have been shown to be activated by ultrasound-induced cavitation. To illustrate this, we demonstrate the activation of a photodynamic therapy agent using microbubbles and ultrasound. Since ultrasound can be accurately focused at large tissue depths, this opens up the potential for generating light at locations that cannot be reached by external sources. This could be exploited both for diagnostic and therapeutic applications, significantly increasing the range of applications that are currently restricted by the limited penetration of light in the tissue.
\end{abstract}

KEYWORDS: sonoluminescence, microbubbles, ultrasound, sonodynamic therapy

\section{INTRODUCTION}

Multibubble sonoluminescence is an intense thermal process whereby transient species formed during the collapse of bubbles under ultrasound (US) excitation (cavitation) emit light. ${ }^{1}$ The majority of previous studies on sonoluminescence have employed ultrasound frequencies and intensities that are significantly different from those used in diagnostic or therapeutic ultrasound. ${ }^{2-7}$ However, with the increasing use of microbubbles (MBs) in both ultrasound imaging and therapy, ${ }^{8}$ and studies showing sonoluminescence at ultrasound frequencies in the megahertz range, ${ }^{9-15}$ there is a need to understand whether these extreme events can, in fact, occur in the tissue.

In particular, sonoluminescence and the reactive oxygen species (ROS) associated with violent bubble collapse have been suggested as the means by which certain classes of drug can be activated by ultrasound, ${ }^{16-20}$ so-called sonodynamic therapy (SDT). Reports on SDT have demonstrated promising results for the treatment of aggressive and resistant tumor cell lines. $^{21-23}$ This approach relies on the combination of ultrasound, ground-state molecular oxygen, and a "sensitizer" drug to produce cytotoxic reactive oxygen species in a targeted manner. Thus, SDT uses a similar approach to photodynamic therapy (PDT), a modality clinically approved for the treatment of superficial lesions and lesions that can be reached with an endoscope. ${ }^{24}$ Ultrasound can, however, be more tightly focused in deeper regions of human tissues compared to light, allowing SDT potentially to treat a wider range of lesions, compared to photodynamic therapy.

The initial findings of drug activation using ultrasound were reported in $1989,{ }^{17}$ and since then, a range of sensitizers have been investigated. ${ }^{22,23}$ Over the last decade, microbubbles have been shown to enhance SDT, and a correlation between SDT and cavitation has been established, but the underlying mechanisms responsible for sensitizer activation have remained uncertain. Several theories have been proposed, including sonoluminescence ${ }^{14,15}$ and pyrolysis, ${ }^{25}$ but a consensus has yet to be drawn. The aim of this study was to investigate whether sonoluminescence events occur during the excitation of phospholipid-coated microbubbles using ultrasound parameters previously shown to have a therapeutic effect in vivo, ${ }^{26-29}$ and whether these events could activate a known SDT sensitizer (Rose Bengal (RB)). Investigation was also made of the production of different types of reactive oxygen species to

Received: April 23, 2019

Accepted: May 10, 2019

Published: May 10, 2019 


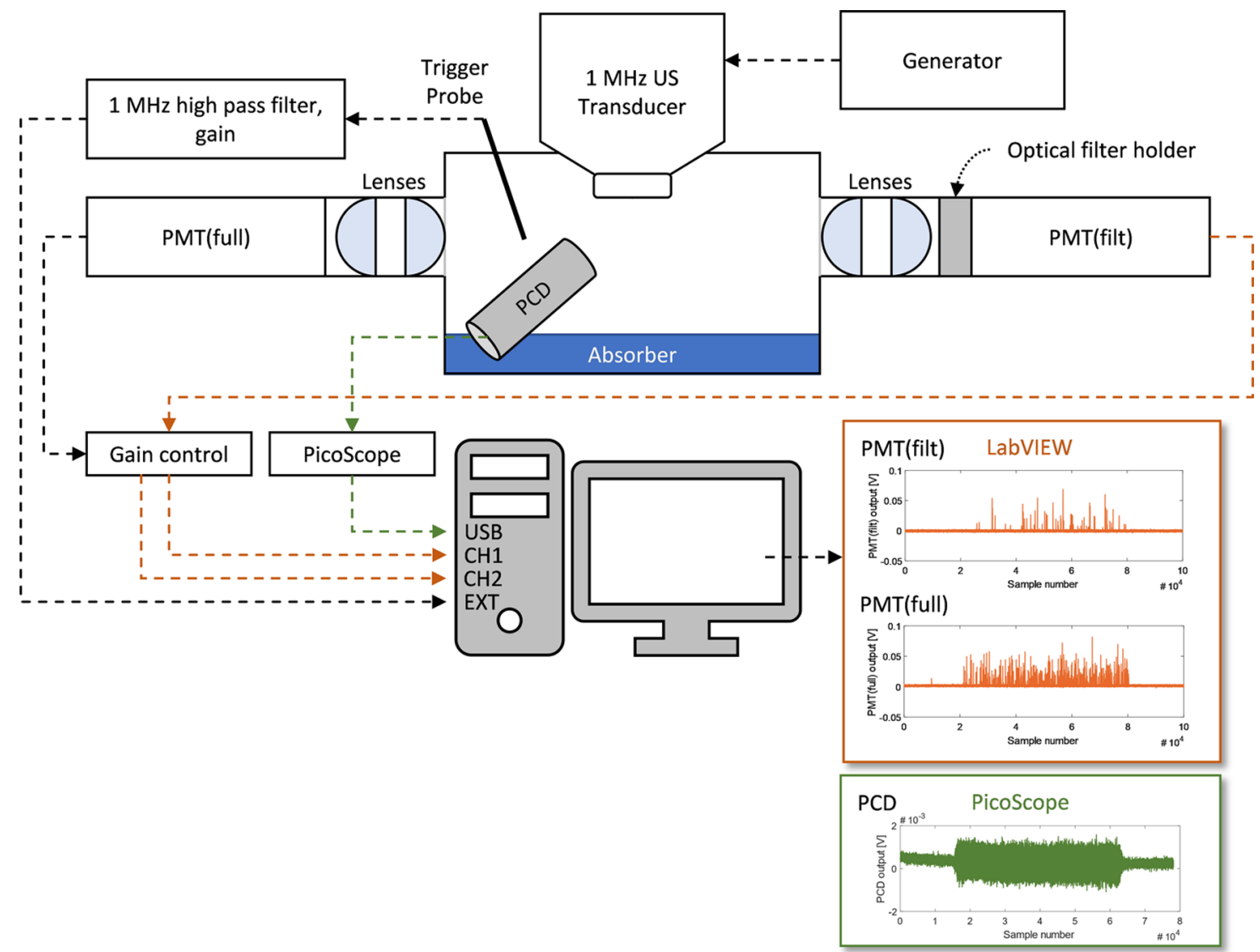

Figure 1. Schematic of the experimental setup used for the simultaneous recording of optical and acoustic emissions.

determine whether their formation could provide an alternative or complementary pathway for sensitizer activation via pyrolysis (please see the Supporting Information).

\section{EXPERIMENTAL SECTION}

Microbubbles. 1,2-Dibehenoyl-sn-glycero-3-phosphocholine (DBPC) was obtained from Avanti Polar Lipids Inc. (Alabaster, Alabama). N-(7-Nitrobenz-2-oxa-1,3-diazol-4-yl)-1,2-dihexadecanoylsn-glycero-3-phosphoethanolamine, 1,3-diphenylisobenzofuran (DPBF), triethylammonium salt, and singlet oxygen sensor green (SOSG) were purchased from Thermo Fisher Scientific. Rose Bengal, polyoxyethylene (40) stearate (PEG $40 \mathrm{~S}$ ), chloroform, and ethanol were all obtained from Sigma-Aldrich Ltd. (Gillingham, Dorset, U.K.). Sulfur hexafluoride $\left(\mathrm{SF}_{6}\right)$ and oxygen $\left(\mathrm{O}_{2}\right)$ gases were purchased from The BOC Group (Guilford, Surrey, U.K.). SonoVue was purchased from Bracco Research (Geneva, Switzerland).

To produce the microbubbles, a mixture of DBPC and PEG40Ss dissolved in chloroform (9:1 molar ratio) was added to a glass vial to produce a $5 \mathrm{~mL}$ batch of microbubbles at a total concentration of 4 $\mathrm{mg} / \mathrm{mL}$. The sample was covered with a pierced parafilm and set on a hot plate at $50{ }^{\circ} \mathrm{C}$ for $12 \mathrm{~h}$ to evaporate the chloroform. Once all of the solvents evaporated, the dried lipid film was suspended in $5 \mathrm{~mL}$ of filtered deionized water for $1 \mathrm{~h}$ at $80{ }^{\circ} \mathrm{C}$ under constant magnetic stirring. The magnetic stir bar was then removed.

The lipid mix solution was sonicated at low intensity (QSonica Q125, $20 \mathrm{kHz}, 3 \mathrm{~mm}$ probe tip, amplitude: $20 \%, 1 \mathrm{~min}$ ) with the sonicator probe tip immersed in the solution. The sonicator probe tip was moved to touch the air and water interface, and a light flow of sulfur hexafluoride $\left(\mathrm{SF}_{6}\right)$ gas was added to fill the headspace of the sample vial. The sonicator was then turned on at high intensity (amplitude: 80\%, $20 \mathrm{~s}$ ). The samples were capped and cooled on ice for $10 \mathrm{~min}$, after which a layer of foam at the top of the sample and a thick layer of densely packed microbubbles underneath the foam were visible. To produce $\mathrm{O}_{2}$-filled microbubbles, a $1 \mathrm{~mL}$ sample of $\mathrm{SF}_{6}$ MBs was sparged with oxygen for $2 \mathrm{~min}$, as described in. ${ }^{30}$ SonoVue was prepared according to the manufacturer's instructions.

Microbubble size and concentration were determined through an analysis of optical images as previous studies have confirmed the reliability of this method compared to particle-sizing devices. ${ }^{31}$ For this, the microbubble suspension was diluted 1:20 in phosphatebuffered saline and $10 \mu \mathrm{L}$ was loaded onto a hemocytometer with a cover slide. Microscope images (30) were acquired through an optical microscope (Leica DM500 optical microscope, Larch House, Milton Keynes, MK14 6FG, U.K.) with a $40 \times$ objective lens at room temperature. The images were then analyzed using a purpose-written MATLAB code (R2016b, The MathWorks, Natick, MA) to determine microbubble mean size and concentration. For all experiments, microbubbles with a modal diameter of $2.1 \pm 1.6 \mu \mathrm{m}$ (Figure S1) were used, corresponding to the agents used in ultrasound imaging and therapy. These were also diluted to $5 \times 10^{5}$ microbubble $/ \mathrm{mL}$ in deionized water to reflect the concentrations that would be present in the human blood stream following injection.

Exposure Chamber. A chamber was designed and built for the characterization of sonoluminescence events to enable simultaneous measurements of photon and acoustic emissions from ultrasoundexcited microbubbles. This consisted of a cube, made of black Delrin to minimize external light contamination, with an internal volume of $100 \mathrm{~mL}$ (Figure S1). The base of the chamber was coated with an ultrasound-absorbing material (F28, Precision Acoustics, Dorset, U.K.) to avoid the formation of standing waves. Ports in the walls enabled coalignment of the foci of two optical lenses (ACL25416U-A, $\varnothing=2.54 \mathrm{~cm}, f=16 \mathrm{~mm}, \mathrm{NA}=0.79$, ThorLabs, Ely, U.K.) and two ultrasound transducers at the center of the chamber. Photons were detected using two photomultiplier tubes (PMTs, Hamamatsu H10493-03, Welwyn Garden City, U.K.) coupled to the lenses. The first ultrasound transducer ( $1 \mathrm{MHz}$ center frequency, $16 \mathrm{~mm}$ element diameter with an integrated drive system, Sonidel SP100, Dublin, 
Ireland) was used to transmit ultrasound to excite the microbubbles. The second transducer $(7.5 \mathrm{MHz}$ center frequency unfocused, element diameter $1.25 \mathrm{~cm}$, Olympus V320, Southend on Sea, U.K.) was used to passively receive nonlinear acoustic emissions indicative of cavitation activity. A schematic of the setup and associated instrumentation is shown in Figure 1.

Experimental Protocol. Sonoluminescence events were investigated in aqueous solutions $\pm \mathrm{MB}, \pm$ Rose Bengal. Samples were prepared in filtered deionized water to obtain $5 \times 10^{5} \mathrm{MB} / \mathrm{mL}$ as above and $2.5 \mu \mathrm{M} \mathrm{RB}$. Samples were injected into the chamber via the filling port and exposed to ultrasound for $2 \mathrm{mins}(1 \mathrm{MHz}$ center frequency, $3.5 \mathrm{~W} / \mathrm{cm} 2$ temporal peak average intensity, $30 \%$ duty cycle, $100 \mathrm{~Hz}$ pulse repetition frequency) during which period 1000 photomultiplier tube (PMT) acquisitions were recorded. The first PMT was used to measure overall light emissions. The second was used with appropriate filters to measure sonoluminescence at specific wavelengths. The corresponding acoustic emissions were recorded using the $7.5 \mathrm{MHz}$ center frequency transducer. A $2 \mathrm{MHz}$ high-pass filter was used to remove the drive frequency from the recorded passive cavitation detector traces before preamplifying (SR445A, SRS, Sunnyvale, CA), digitizing it (Handyscope HS3, TiePie Engineering, Sneek, Netherlands), and saving it on a computer drive for analysis. The effect of bulk temperature was examined to determine if sonoluminescence could occur at biologically relevant temperatures. For this, experiments were conducted with a sample temperature of 10,23 , and $37{ }^{\circ} \mathrm{C}$, monitored using a PCE-T390 digital thermometer from PCE Instruments, before and after ultrasound exposure.

Detection of Reactive Oxygen Species. The detection of singlet oxygen specifically was accomplished using the commercial product: singlet oxygen sensor green (SOSG), which reacts with ${ }^{1} \mathrm{O}_{2}$ to form SOSG-endoperoxides with a strong fluorescence emission of around $525-536 \mathrm{~nm}$. The less specific detection of both ${ }^{1} \mathrm{O}_{2}$ and/or $\mathrm{O}_{2}{ }^{\bullet}$ was determined through a decrease in the absorbance of 1,3diphenylisobenzofuran (DPBF) at $410 \mathrm{~nm}$ as it oxidizes in the presence of either species forming nonfluorescent 1,2-phenylenebis(phenylmethanone). For the detection of hydroxyl radical, nonfluorescent benzoic acid was used as it becomes permanently fluorescent (Ex: $305 \mathrm{~nm} / \mathrm{Em}: 420 \mathrm{~nm}$ ) upon aromatic hydroxylation by ${ }^{\circ} \mathrm{OH}$.

Fluorescence and absorbance measurements were done in quadruplets on COSTAR or Greigner UV-Star clear flat-bottom 96well plates from Sigma-Aldrich (Dorset, U.K.), using a FLUOstar Omega multipurpose plate reader from BMG Labtech (Aylesbury, Bucks, U.K.) at room temperature. For some of the examination, these measurements were taken before and after sample exposition to determine a percent change in the intensity relative to the preexposure intensity. Sample absorbance measurements were all normalized to that of blank control.

Data Analysis. The acquired acoustic emission traces were fitted with a Tukey window to avoid discontinuities and then analyzed with a fast Fourier transform using MATLAB (R2017b The Mathworks, Natick, MA). The harmonics (multiples of the drive frequency \pm 100 $\mathrm{kHz},>2 \mathrm{MHz}$ ), ultraharmonics (half-integer harmonics of the drive frequency $\pm 50 \mathrm{kHz},>2 \mathrm{MHz}$ ), and broad-band (remaining signal $>2$ $\mathrm{MHz}$ ) components were extracted for each acquisition. The power and cumulative energy in these frequency subsets were calculated for each acquisition over the entire exposure time. To characterize the spectrum of the sonoluminescence, the signal of the filtered PMT was normalized with the total amount of light generated (Figure S1). This enabled a comparison between experimental runs. Each experiment was repeated $n=3$ times. The fluorescence and absorbance readings were also performed four times for each sample. The average and the standard deviation within each group are presented.

\section{RESULTS AND DISCUSSION}

Measurements of sonoluminescence and acoustic emissions at $23{ }^{\circ} \mathrm{C}$ were made for microbubbles manufactured in-house ${ }^{28}$ with a sulfur hexafluoride $\left(\mathrm{SF}_{6}\right)$ or oxygen $\left(\mathrm{O}_{2}\right)$ gas cores and the commercially available contrast agent Sonovue (Figure
S1). While all microbubbles tested produced sonoluminescence when exposed to US (Figure 2), reduced sonolumi-

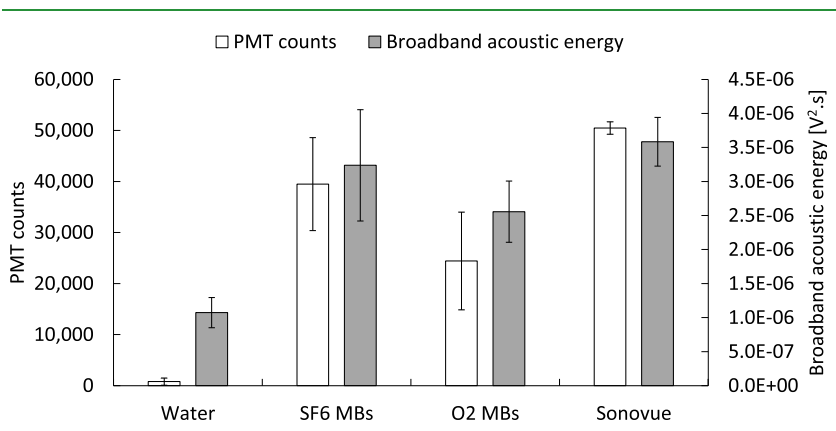

Figure 2. Sonoluminescence and broad-band acoustic emissions produced by phospholipid-coated microbubbles driven at $1 \mathrm{MHz}$ with an intensity of $3.5 \mathrm{~W} / \mathrm{cm}^{2}$, a $30 \%$ duty cycle, and a $100 \mathrm{~Hz}$ pulse repetition frequency for $2 \mathrm{~min}$. The total photomultiplier tube (PMT) counts above $6 \mathrm{mV}$ in amplitude and broad-band energy of acoustic emissions for three microbubble formulations and water control are displayed ( $n=3$ runs of 1000 acquisitions each, and error bars indicate standard deviations).

nescence counts were observed for $\mathrm{O}_{2}$ microbubbles compared to Sonovue and $\mathrm{SF}_{6}$ microbubbles. This was attributed to the lower stability of $\mathrm{O}_{2}$ microbubbles and the higher solubility of $\mathrm{O}_{2}$ in aqueous solutions compared to those of $\mathrm{SF}_{6}$. The reduced broad-band energy levels produced by $\mathrm{O}_{2}$ microbubbles (Figure 2) further confirmed these results. The pulse height distribution of individual sonoluminescence events was, however, found to be comparable between the formulations (Figure S2), highlighting that the cavitation of these systems generated comparable collapse conditions and sonoluminescence. $^{10,32}$

The spectrum of the light generated using $\mathrm{SF}_{6}$ microbubbles was measured using a set of five optical filters at room temperature. Figure 3 shows a broad spectrum with an increased sonoluminescence generation at the lower wavelengths. As the intensity of sonoluminescence reported here was low, the use of a monochromator to obtain a higher-

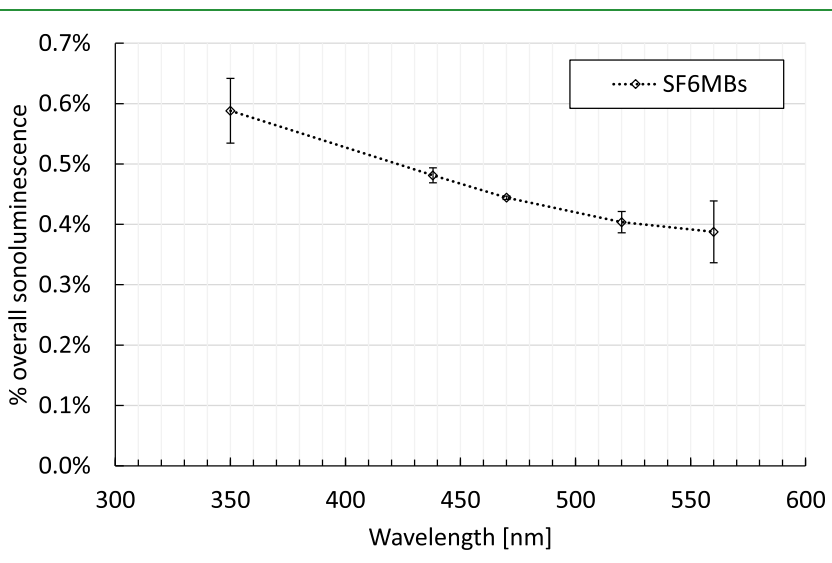

Figure 3. Spectrum of sonoluminescence for diluted $\mathrm{SF}_{6}$ microbubbles at $23{ }^{\circ} \mathrm{C}$. The percentage of overall sonoluminescence reflects the normalized counts from the filtered PMT at specific wavelengths over 1000 acquisitions with the counts from a nonfiltered PMT. The normalized count was then corrected for the bandwidth of the optical filters used and the PMT sensitivity at that wavelength. $n=3$ runs were performed for each wavelength, and error bars indicate the standard deviation between the runs. 
(a) Total PMT count $>6 \mathrm{mV}$

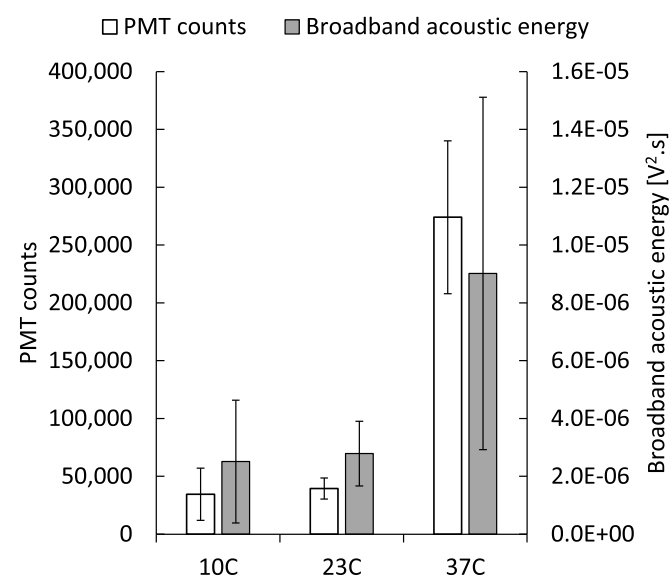

(b) PMT signal height distribution

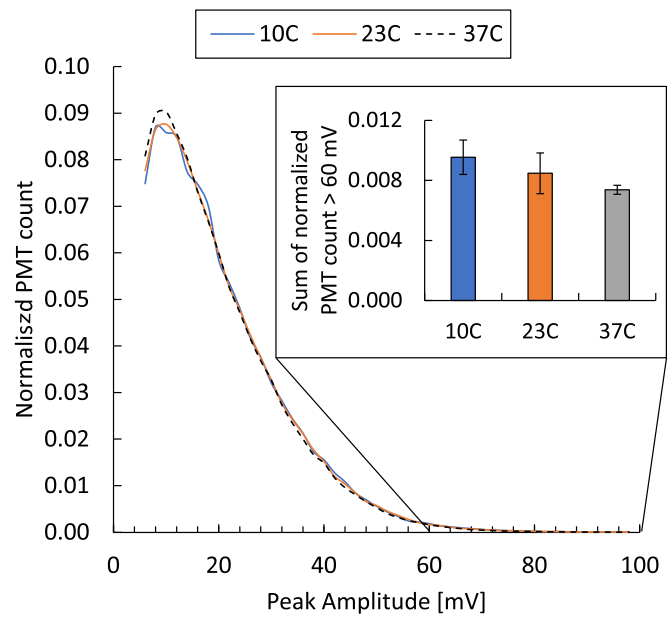

Figure 4. Sonoluminescence and broad-band acoustic emissions from $\mathrm{SF}_{6}$ microbubbles diluted in deionized water at 10,23 , and $37{ }^{\circ} \mathrm{C}(n=3$, error bars indicate standard deviations for each run). (a) Total PMT counts above $6 \mathrm{mV}$ in amplitude and broad-band energy for three different temperatures are displayed. (b) Number of PMT counts for increasing peak amplitude. Number of PMT counts in each bin is normalized by the total number of counts recorded in each run. The inset shows the sum of normalized PMT counts above $60 \mathrm{mV}$ for each temperature tested. These results indicate that with increasing temperatures, while the number of cavitation events increases, their amplitude decreases. The sample temperature before and after ultrasound exposure did not fluctuate substantially $\left( \pm 1^{\circ} \mathrm{C}\right)$.

wavelength resolution was not feasible, and thus specific molecular features in the optical spectrum were not discernible. However, sonoluminescence in water has been reported at 1 $\mathrm{MHz}$ with a broad continuous spectrum and no molecular features. $^{12,33,34}$ Such broad spectra have been associated with radiation emissions from cavitation events, e.g., blackbody, ${ }^{9}$ bremsstrahlung, ${ }^{35,36}$ and/or recombination radiations. ${ }^{37,38}$ Although no consensus has been reached on the exact mechanisms, the generation of reactive oxygen species (ROS) during microbubble cavitation such as hydrogen peroxide $^{39}$ and hydroxyl radical ${ }^{40,41}$ concurs with the recombination radiation theory. ${ }^{12,38}$

An increase in bulk solution temperature has been reported to affect sonoluminescence by (1) increasing the number of sonoluminescence events due to an increase in the number of cavitation events ${ }^{10}$ and (2) lowering the amplitude of individual sonoluminescence events due to lowering of the intensity of collapse. ${ }^{32}$ Measurements were taken at 10, 23, and $37^{\circ}$, and their comparison in Figure $4 \mathrm{a}$ shows the expected increase in sonoluminescence occurrence and broad-band activity at $37{ }^{\circ} \mathrm{C}$ when $\mathrm{SF}_{6}$ microbubbles were used as cavitation nuclei. As the amplitude of sonoluminescence was examined (Figure 4b), a decrease in the number of highamplitude sonoluminescence events $(>60 \mathrm{mV})$ was observed with higher bulk solution temperatures (Figure $4 \mathrm{~b}$ inset). Thus, these results demonstrate that at biologically relevant temperatures, a greater number of sonoluminescence events occur when microbubbles are exposed to mild therapeutic US conditions; however, the amplitude of individual sonoluminescence events is reduced.

The generation of sonoluminescence by microbubbles and ultrasound is potentially of great importance for the fields of both photo- and sonodynamic therapies (PDT and SDT, respectively). In PDT, significant efforts have been made to design sensitizers with increased absorption at wavelengths that allow improved penetration of light in the tissue. Although the therapeutic effects of SDT have been reported since $1989,{ }^{17}$ the explanation behind the activation of the sensitizer with this method was not well accepted. Therefore, we measured the sonoluminescence output with and without the presence of an absorbing sensitizer, in this case, Rose Bengal $(\mathrm{RB})$. Figure 5a highlights that the presence of $\mathrm{RB}$ and $\mathrm{SF}_{6}$ microbubbles at $37{ }^{\circ} \mathrm{C}$ led to a decrease in sonoluminescence measured at the absorption wavelength of the drug $(560 \mathrm{~nm}$, Figure S3) compared to a reference wavelength $(350 \mathrm{~nm})$. Hence, sonoluminescence from cavitating microbubbles can be absorbed by the surrounding sensitizers, leading to their activation. These results support the hypothesis that sonoluminescence and the resulting transfer of energy to an accepting sensitizer are a key mechanism underlying SDT and are consistent with the reports by Umemura et al. ${ }^{15}$ and Giuntini et al. ${ }^{14}$ at room temperatures and without the use of exogenously added cavitation nuclei. Additionally, Figure $5 \mathrm{~b}$ shows that at the same ultrasound parameters, the combination of $\mathrm{RB}$ and $\mathrm{SF}_{6}$ microbubbles in solution produced significantly more singlet oxygen radicals compared to that of microbubbles alone, confirming the activation of RB.

In contrast, the presence of reactive oxygen species did not affect the activity of Rose Bengal at ambient temperatures and pressures (Figure S4), indicating that pyrolysis-induced ROS generation is not involved in the activation of Rose Bengal. Further, microbubble cavitation did not lead to significant degradation of Rose Bengal, demonstrating that significant pyrolysis of the sensitizer itself does not occur at these exposure conditions (Figure S5).

There are several aspects of these results that may be important for both diagnostic and therapeutic applications of ultrasound and microbubbles. In the absence of cavitation, ultrasound is a nonionizing modality, and epidemiological studies of ultrasound imaging have not identified any significant health hazards associated with the technique. ${ }^{42,43}$ Yet, bubble cavitation was shown to cause ionization of molecules as seen with a broad continuum of sonoluminescence, and the production of excited species and radicals. ${ }^{36,38}$ Here, we demonstrate that, in the presence of microbubbles, cavitation produces reactive oxygen species and sonolumines- 

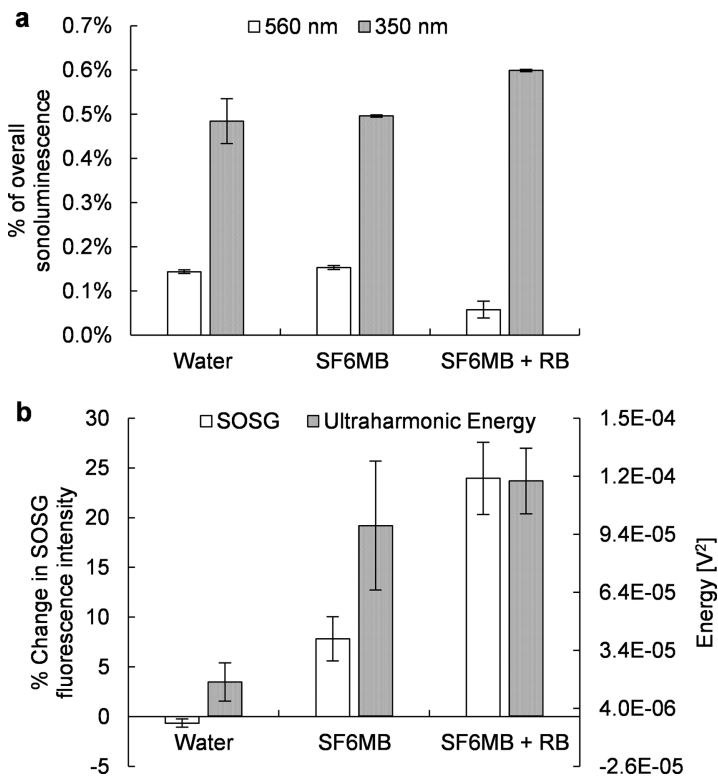

Figure 5. Addition of Rose Bengal (RB) to $\mathrm{SF}_{6}$ microbubbles $\left(\mathrm{SF}_{6}\right.$ $\mathrm{MB}$ ), and the resulting effect on sonoluminescence, broad-band emissions, and singlet oxygen radical generation. (a) Percent of overall sonoluminescence measured with optical filters for 560 and $350 \mathrm{~nm}$. Filtered PMT signal was normalized by the overall counts recorded by the nonfiltered reference PMT and for the bandwidth of the filter used. These were acquired for water, $\mathrm{SF}_{6}$ microbubbles $\left(\mathrm{SF}_{6}\right.$ $\mathrm{MB}), \mathrm{SF}_{6}$ microbubbles, and Rose Bengal samples at $37^{\circ} \mathrm{C}(n=3$ runs, each of 1000 acquisitions). Rose Bengal peak absorbance is known to be at $559 \mathrm{~nm}$ (Figure S3). The sonoluminescence measurement at $560 \mathrm{~nm}$ was made to assess the absorption of sonoluminescence by the sensitizer and compared to $350 \mathrm{~nm}$ for reference. (b) Activation of Rose Bengal was assessed through the generation of cytotoxic singlet oxygen. This was characterized by an increase in the fluorescence intensity of singlet oxygen sensor green (SOSG, left axis). The different groups were exposed to $1 \mathrm{MHz}, 462$ $\mathrm{mV}_{\mathrm{pk}-\mathrm{pk}} 30 \%$ duty cycles, and a $100 \mathrm{~Hz}$ pulse repetition frequency for $30 \mathrm{~s}(n=3)$. The ultraharmonic emissions of microbubbles were captured using a passive acoustic detector and displayed as the overall ultraharmonic energy during the exposure (right axis).

cence. The sonoluminescence measured in this study is unlikely to cause phototoxicity as the number of photons is below that associated with the safe use of lasers in medical applications. ${ }^{44,45}$ In contrast, the generation of free radicals could cause local cytotoxicity, although the short lifetimes of radicals $^{46}$ and the small reaction volume ${ }^{1}$ of cavitation will restrict the region of damage, making such an approach ideal for targeted applications in oncology.

\section{CONCLUSIONS}

In summary, the cavitation of microbubbles under mild therapeutic ultrasound conditions was found to generate sonoluminescence, the intensity of which was positively correlated with the broad-band energy of microbubble acoustic emissions. Further, this work confirms that sonoluminescence is involved in the activation of photosensitizers, which allows greater production of reactive oxygen species during SDT. The sensitizers used for PDT can then be locally activated by energy transfer through the sonoluminescence generated by ultrasound and microbubbles, enabling the treatment of a wider range of lesions using SDT.

\section{ASSOCIATED CONTENT}

\section{Supporting Information}

The Supporting Information is available free of charge on the ACS Publications website at DOI: 10.1021/acsami.9b07084.

Simultaneous sonoluminescence and acoustic emissions recording from microbubbles dispersed in aqueous solution (Figure S1); comparison of sonoluminescence amplitude for $\mathrm{SF}_{6}, \mathrm{O}_{2}$, and Sonovue microbubbles (Figure S2); absorbance spectrum of Rose Bengal in an aqueous solution of phospholipids (Figure S3); activation of Rose Bengal by different reactive oxygen species (Figure S4); absorbance of aqueous Rose Bengal solutions at $559 \mathrm{~nm}$ (Figure S5); and summary for the samples tested for the production of ROS from Rose Bengal (Tables S1 and S2) (PDF)

\section{AUTHOR INFORMATION}

\section{Corresponding Authors}

*E-mail: ap.mchale@ulster.ac.uk. Phone: +44(0)28 70124616 (A.P.M.).

*E-mail: j.callan@ulster.ac.uk. Phone: +44(0)28 70123059 (J.F.C.).

*E-mail: eleanor.stride@eng.ox.ac.uk. Phone: +44(0) 1865617747 (E.S.).

\section{ORCID}

Shamit Shrivastava: 0000-0003-0916-7336

Anthony P. McHale: 0000-0003-2397-648X

Eleanor Stride: 0000-0003-3371-5929

\section{Notes}

The authors declare no competing financial interest. The raw data are available from the Oxford University Research Archive ora.ox.ac.uk.

\section{ACKNOWLEDGMENTS}

The authors thank the Engineering and Physical Sciences Research Council for support through grants EP/I021795/1 and EP/L024012/1. J.F.C. thanks Norbrook Laboratories Ltd. for an endowed chair. E.B. thanks the Research Councils UK Digital Economy Programme for support through grant EP/ G036861/1 (Oxford Centre for Doctoral Training in Healthcare Innovation).

\section{REFERENCES}

(1) Suslick, K. S.; Doktycz, S. J.; Flint, E. B. On the Origin of Sonoluminescence and Sonochemistry. Ultrasonics 1990, 28, 280290.

(2) Suslick, K. S.; Didenko, Y.; Fang, M. M.; Hyeon, T.; Kolbeck, K. J.; McNamara, W. B., III; Mdleleni, M. M.; Wong, M. Acoustic Cavitation and Its Consequences. Philos. Trans. R. Soc., A 1999, 357, 335-353.

(3) McNamara, W. B.; Didenko, Y. T.; Suslick, K. S. Sonoluminescence Temperatures during Multi-Bubble Cavitation. Nature 1999, 401, 772-775.

(4) Lepoint-Mullie, F.; Voglet, N.; Lepoint, T.; Avni, R. Evidence for the Emission of "alkali-Metal-Noble-Gas" van Der Waals Molecules from Cavitation Bubbles. Ultrason. Sonochem. 2001, 8, 151-158.

(5) Vazquez, G.; Camara, C.; Putterman, S.; Weninger, K. Sonoluminescence: Nature's Smallest Blackbody. Opt. Lett. 2001, 26, 575-577.

(6) Brenner, M. P.; et al. Single-Bubble Sonoluminescence. Rev. Mod. Phys. 2002, 74, 425-484.

(7) Miller, D. L.; Smith, N. B.; Bailey, M. R.; Czarnota, G. J.; Hynynen, K.; Makin, I. R. S. Overview of Therapeutic Ultrasound 
Applications and Safety Considerations. J. Ultrasound Med. 2012, 31, 623-634.

(8) Stride, E.; Saffari, N. Microbubble Ultrasound Contrast Agents: A Review. Proc. Inst. Mech. Eng., Part H 2003, 217, 429-447.

(9) Srinivasan, D.; Holroyd, L. V. Optical Spectrum of the Sonoluminescence Emitted by Cavitated Water. J. Appl. Phys. 1961, 32, 446-453.

(10) Pickworth, M. J. W.; Dendy, P. P.; Leighton, T. G.; Walton, A. J. Studies of the Cavitational Effects of Clinical Ultrasound by Sonoluminescence: 2. Thresholds for Sonoluminescence from a Therapeutic Ultrasound Beam and the Effect of Temperature and Duty Cycle. Phys. Med. Biol. 1988, 33, 1249-1260.

(11) Ciuti, P.; Dezhkunov, N. V.; Iernetti, G.; Kulak, A. I. Cavitation Phenomena in Pulse Modulated Ultrasound Fields. Ultrasonics 1998, 36, 569-574.

(12) Beckett, M. A.; Hua, I. Impact of Ultrasonic Frequency on Aqueous Sonoluminescence and Sonochemistry. J. Phys. Chem. A 2001, 105, 3796-3802.

(13) Weninger, K. R.; Camara, C. G.; Putterman, S. J. Observation of Bubble Dynamics within Luminescent Cavitation Clouds: Sonoluminescence at the Nano-Scale. Phys. Rev. E 2001, 63, No. 016310 .

(14) Giuntini, F.; Foglietta, F.; Marucco, A. M.; Troia, A.; Dezhkunov, N. V.; Pozzoli, A.; Durando, G.; Fenoglio, I.; Serpe, L.; Canaparo, R. Insight into Ultrasound-Mediated Reactive Oxygen Species Generation by Various Metal-Porphyrin Complexes. Free Radical Biol. Med. 2018, 121, 190-201.

(15) Umemura, S.; Yumita, N.; Nishigaki, R.; Umemura, K. Mechanism of Cell Damage by Ultrasound in Combination with Hematoporphyrin. Jpn. J. Cancer Res. 1990, 81, 962-966.

(16) Hachimine, K.; Shibaguchi, H.; Kuroki, M.; Yamada, H.; Kinugasa, T.; Nakae, Y.; Asano, R.; Sakata, I.; Yamashita, Y.; Shirakusa, T.; Kuroki, M. Sonodynamic Therapy of Cancer Using a Novel Porphyrin Derivative, DCPH-P-Na(I), Which Is Devoid of Photosensitivity. Cancer Sci. 2007, 98, 916-920.

(17) Yumita, N.; Nishigaki, R.; Umemura, K.; Umemura, S. -i Hematoporphyrin as a Sensitizer of Cell-damaging Effect of Ultrasound. Jpn. J. Cancer Res. 1989, 219-222.

(18) Ninomiya, K.; Noda, K.; Ogino, C.; Kuroda, S. I.; Shimizu, N. Enhanced $\mathrm{OH}$ Radical Generation by Dual-Frequency Ultrasound with TiO 2 Nanoparticles: Its Application to Targeted Sonodynamic Therapy. Ultrason. Sonochem. 2014, 21, 289-294.

(19) Osaki, T.; Yokoe, I.; Uto, Y.; Ishizuka, M.; Tanaka, T.; Yamanaka, N.; Kurahashi, T.; Azuma, K.; Murahata, Y.; Tsuka, T.; Ito, N.; Imagawa, T.; Okamoto, Y. Bleomycin Enhances the Efficacy of Sonodynamic Therapy Using Aluminum Phthalocyanine Disulfonate. Ultrason. Sonochem. 2016, 28, 161-168.

(20) Sakusabe, N.; Okada, K.; Sato, K.; Kamada, S.; Yoshida, Y.; Suzuki, T. Enhanced Sonodynamic Antitumor Effect of Ultrasound in the Presence of Nonsteroidal Anti-Inflammatory Drugs. Jpn. J. Cancer Res. 1999, 90, 1146-1151.

(21) Chen, H.; Zhou, X.; Gao, Y.; Zheng, B.; Tang, F.; Huang, J. Recent Progress in Development of New Sonosensitizers for Sonodynamic Cancer Therapy. Drug Discovery Today 2014, 502-509.

(22) Trendowski, M. Using the Promise of Sonodynamic Therapy in the Clinical Setting against Disseminated. Chemother. Res. Pract. 2015, 2015, 1-16.

(23) Costley, D.; Mc Ewan, C.; Fowley, C.; McHale, A. P.; Atchison, J.; Nomikou, N.; Callan, J. F. Treating Cancer with Sonodynamic Therapy: A Review. Int. J. Hyperthermia 2015, 107-117.

(24) Dolmans, D.; Fukumura, D.; Jain, R. K. TIMELINE: Photodynamic Therapy for Cancer. Nat. Rev. Cancer 2003, 3, 380387.

(25) Mišík, V.; Riesz, P. Free Radical Intermediates in Sonodynamic Therapy. Ann. N. Y. Acad. Sci. 2000, 899, 335-348.

(26) Nomikou, N.; Fowley, C.; Byrne, N. M.; McCaughan, B.; McHale, A. P.; Callan, J. F. Microbubble-Sonosensitiser Conjugates as Therapeutics in Sonodynamic Therapy. Chem. Commun. 2012, 48, $8332-8334$
(27) Chen, H.; Hwang, J. H. Ultrasound-Targeted Microbubble Destruction for Chemotherapeutic Drug Delivery to Solid Tumors. J. Ther. Ultrasound 2013, 1, No. 10.

(28) McEwan, C.; Owen, J.; Stride, E.; Fowley, C.; Nesbitt, H.; Cochrane, D.; Coussios, C. C.; Borden, M.; Nomikou, N.; McHale, A. P.; Callan, J. F. Oxygen Carrying Microbubbles for Enhanced Sonodynamic Therapy of Hypoxic Tumours. J. Controlled Release 2015, 203, 51-56.

(29) Sheng, Y.; Beguin, E.; Nesbitt, H.; Kamila, S.; Owen, J.; Barnsley, L. C.; Callan, B.; O’Kane, C.; Nomikou, N.; Hamoudi, R.; Taylor, M. A.; Love, M.; Kelly, P.; O’Rourke, D.; Stride, E.; McHale, A. P.; Callan, J. F. Magnetically Responsive Microbubbles as Delivery Vehicles for Targeted Sonodynamic and Antimetabolite Therapy of Pancreatic Cancer. J. Controlled Release 2017, 262, 192-200.

(30) McEwan, C.; Owen, J.; Stride, E.; Fowley, C.; Nesbitt, H.; Cochrane, D.; Coussios, C. C.; Borden, M.; Nomikou, N.; McHale, A. P.; Callan, J. F. Oxygen Carrying Microbubbles for Enhanced Sonodynamic Therapy of Hypoxic Tumours. J. Controlled Release 2015, 203, 51-56.

(31) Sennoga, C. A.; Mahue, V.; Loughran, J.; Casey, J.; Seddon, J. M.; Tang, M.; Eckersley, R. J. On Sizing and Counting of Microbubbles Using Optical. Ultrasound Med. Biol. 2010, 36, 20932096.

(32) Hilgenfeldt, S.; Lohse, D.; Moss, W. C. Water Temperature Dependence of Single Bubble Sonoluminescence. Phys. Rev. Lett. 1998, 80, 1332-1335.

(33) Choi, P. K.; Fuayama, K. Multibubble Sonoluminescence and $\mathrm{Na}$ Atom Emission in Sodium Dodecyl Sulfate Surfactant Solutions. Jpn. J. Appl. Phys. 2007, 46, 4768-4770.

(34) Didenko, Y. T.; Nastich, D. N.; Pugach, S. P.; Polovinka, Y. A.; Kvochka, V. I. The Effect of Bulk Solution Temperature on the Intensity and Spectra of Water Sonoluminescence. Ultrasonics 1994, $32,71-76$.

(35) Moss, W. C.; Clarke, D. B.; Young, D. A. Calculated Pulse Widths and Spectra of a Single Sonoluminescing Bubble. Science 1997, 276, 1398-1401.

(36) Bernstein, L. S.; Zakin, M. R.; Flint, E. B.; Suslick, K. S. Cavitation Thermometry Using Molecular and Continuum Sonoluminescence. J. Acoust. Soc. Am. 1996, 100, 2679.

(37) Hilgenfeldt, S.; Grossmann, S.; Lohse, D. A Simple Explanation of Light Emission in Sonoluminescence. Nature 1999, 398, 402-405.

(38) Sehgal, C.; Sutherland, R. G.; Verrall, R. E. Optical Spectra of Sonoluminescence from Transient and Stable Cavitation in Water Saturated with Various Gases. J. Phys. Chem. 1980, 84, 388-395.

(39) Juffermans, L. J. M.; Dijkmans, P. A.; Musters, R. J. P.; Visser, C. A.; Kamp, O. Transient Permeabilization of Cell Membranes by Ultrasound-Exposed Microbubbles Is Related to Formation of Hydrogen Peroxide. Am. J. Physiol.: Heart Circ. Physiol. 2006, 291, H1595-H1601.

(40) Escoffre, J.; Campomanes, P.; Tarek, M.; Bouakaz, A. In New Insights in the Role of Reactive Oxygen Species in Mechanisms of Sonoporation: In-Vitro Validation and Molecular Dynamic Simulations, IEEE International Ultrasonics Symposium Proceedings, 2017.

(41) Barati, A. H.; Mokhtari-Dizaji, M.; Mozdarani, H.; Bathaei, S. Z.; Hassan, Z. M. Free Hydroxyl Radical Dosimetry by Using $1 \mathrm{MHz}$ Low Level Ultrasound Waves. Iran. J. Radiat. Res. 2006, 3, 163-169.

(42) Ziskin, M. C.; Petitti, D. B. Epidemiology of Human Exposure to Ultrasound: A Critical Review. Ultrasound Med. Biol. 1988, 14, 9196.

(43) Salvesen, K. Å. EFSUMB: Safety Tutorial: Epidemiology of Diagnostic Ultrasound Exposure during Pregnancy - European Committee for Medical Ultrasound Safety (ECMUS). Eur. J. Ultrasound 2002, 15, 165-171.

(44) International Commission on Non-Ionizing Radiation Protection. ICNIRP Guidelines on Limits of Exposure to Laser Radiation of Wavelengths between $180 \mathrm{Nm}$ and 1,000 Mm. Health Phys. 2013, 105, 271-295.

(45) Tirlapur, U. K.; König, K. Targeted Transfection by Femtosecond Laser. Nature 2002, 418, 290-291. 
(46) Riesz, P.; Kondo, T. Free Radical Formation Induced by Ultrasound and Its Biological Implications. Free Radicals Biol. Med. 1992, 247-270. 